\title{
EXPLICIT REPRESENTATIONS OF THE INTEGRAL CONTAINING THE ERROR TERM IN THE DIVISOR PROBLEM II
}

\author{
JUN FURUYA \\ Department of Integrated Arts and Science, Okinawa National College of Technology, \\ Nago, Okinawa, 905-2192, Japan \\ e-mail: jfuruya@okinawa-ct.ac.jp \\ and YOSHIO TANIGAWA* \\ Graduate School of Mathematics, Nagoya University, Nagoya, 464-8602, Japan \\ e-mail: tanigawa@math.nagoya-u.ac.jp
}

(Received 28 June 2010; revised 10 December 2010; accepted 15 August 2011)

\begin{abstract}
In our previous paper [2], we derived an explicit representation of the integral $\int_{1}^{\infty} t^{-\theta} \Delta(t) \log ^{j} t d t$ by differentiation under the integral sign. Here, $j$ is a fixed natural number, $\theta$ is a complex number with $1<\Re \theta \leq 5 / 4$ and $\Delta(x)$ denotes the error term in the Dirichlet divisor problem. In this paper, we shall reconsider the same formula by an alternative approach, which appeals to only the elementary integral formulas concerning the Riemann zeta- and periodic Bernoulli functions. We also study the corresponding formula in the case of the circle problem of Gauss.
\end{abstract}

2000 Mathematics Subject Classification. 11N37

1. Introduction and statement of results. In the previous paper [2], the authors studied the properties concerning the integral

$$
\int_{1}^{\infty} t^{-\theta} \Delta_{k}(t) \log ^{j} t d t
$$

for a non-negative integer $j$ and a complex number $\theta$, where $\Delta_{k}(x)$ denotes the error term in the divisor problem of Dirichlet and Piltz defined by

$$
\Delta_{k}(x)=\sum_{n \leq x} d_{k}(n)-x \sum_{m=0}^{k-1} a_{m}^{(k)} \log ^{m} x .
$$

Here $d_{k}(n)$ is the number of ways to write the natural number $n$ as the product of $k$ factors, and $a_{m}^{(k)}$ are certain constants. As to the previous works concerning the properties of (1.1), the special value and the explicit representation of this integral in the case $j=0$ have been obtained by Lavrik-Israilov-Edgorov [6] and Sitaramachandrarao [7]. In particular, they studied several types of the representations for the special value of

*The second author is supported by Grant-in-Aid for Scientific Research No. 21540012. 
this integral at $\theta=2$, which correspond to the constant term of the Laurent expansion of $\zeta(s) / s$ at $s=1$. Here $\zeta(s)$ denotes the Riemann zeta-function.

Furthermore, Sitaramachandrarao [7] considered the analytical continuation of this integral by regarding it as a complex function with respect to $\theta$, especially the following formula was derived:

$$
\int_{1}^{\infty} t^{-\theta} \Delta_{k}(t) d t=\frac{1}{\theta-1} \zeta(\theta-1)^{k}-\sum_{m=0}^{k-1} m ! a_{m}^{(k)}\left(\frac{1}{\theta-2}\right)^{m+1},
$$

which is valid (at least) for $\Re \theta>2-1 / k$. In view of the right-hand side in (1.2), we can see that the function

$$
I_{k}(\theta)=\int_{1}^{\infty} t^{-\theta} \Delta_{k}(t) d t
$$

which is originally defined in the region $\Re \theta>2-1 / k$, can be continued analytically to the whole $\theta$-plane as a meromorphic function. This is a holomorphic function except for a simple pole at $\theta=1$. (Note that the point $\theta=2$ does not give a pole of $I_{k}(\theta)$.)

In [2], we studied the properties of (1.1) for $j \geq 0$, especially we treated in detail the case $k=2$, that is, the case of the Dirichlet divisor problem. In this case $\Delta_{2}(x)=\Delta(x)$ is written as

$$
\Delta(x)=\sum_{n \leq x} d(n)-x(\log x+2 \gamma-1)
$$

with the divisor function $d(n)$ and the Euler constant $\gamma$. By the elementary calculations, we see that integral

$$
\int_{1}^{\infty} t^{-\theta} \Delta(t) \log ^{j} t d t
$$

is convergent absolutely and uniformly for every compact subset in $\Re \theta>5 / 4$, and it is divergent for $\Re \theta \leq 1$. We note that the range $\Re \theta>5 / 4$ is slightly better than that mentioned in Sitaramachandrarao's paper [7]. However, we stress that the constant ' $5 / 4$ ' is the best-possible one that we can obtain by the method used in paper [2], since $\Delta(x)=\Omega\left(x^{1 / 4}\right)$, and it seems that integral (1.3) would not be convergent absolutely for $1<\mathfrak{R} \theta \leq 5 / 4$ (cf. Conjecture 1 in [2]). This conjecture means that $t^{-\theta} \Delta(t) \log ^{j} t$ would not be Lebesgue-integrable for $1<\Re \theta \leq 5 / 4$.

In the range $\Re \theta>5 / 4$, we easily see that integral (1.3) can be written down explicitly as

$$
\begin{aligned}
\int_{1}^{\infty} t^{-\theta} \Delta(t) \log ^{j} t d t= & (-1)^{j} \frac{d^{j}}{d \theta^{j}} \int_{1}^{\infty} t^{-\theta} \Delta(t) d t \\
= & \sum_{m=0}^{j}\left(\begin{array}{c}
j \\
m
\end{array}\right) \frac{m !(-1)^{j-m}}{(\theta-1)^{m+1}}\left(\zeta^{2}(\theta-1)\right)^{(j-m)}-\frac{2 \gamma j !}{(\theta-2)^{j+1}} \\
& +\frac{j !(\theta-j-3)}{(\theta-2)^{j+2}} .
\end{aligned}
$$

Since the integrands are Lebesgue-integrable for $\Re \theta>5 / 4$, the interchanging of differentiation and integration in (1.4) can be justified by the Lebesgue integral theory 
(cf. e.g. Theorem 10.39 in [1]). We shall stress that we can say that this expression is a 'trivial formula' regarding integral (1.3) as the complex function $I_{2}(\theta)$. However, our aim of this study, which is the most important point, is that we consider the explicit representation of this function as the 'integral form', not a form of an analytical continuation of this integral.

As with the case $1<\Re \theta \leq 5 / 4$, we studied convergence properties of (1.3) and the explicit representation of this integral in the previous paper [2], and obtained the following theorem.

THEOREM 1 (Theorem 1 in [2]). The integral (1.3) is convergent uniformly on every compact set in the region $1<\Re \theta \leq 5 / 4$, and this can also be expressed by the right-hand side in (1.5) in the same region.

The second assertion of Theorem 1 can be proved from the first one by differentiation under the integral sign. A theorem of such interchange of integration and differentiation of the form

$$
\frac{d}{d x} \int_{1}^{\infty} f(t, x) d t=\int_{1}^{\infty} \frac{\partial}{\partial x} f(t, x) d t
$$

can be found in, for example, Theorem 3.4 (p. 340) in [5], which is stated under the assumption that $f(t, x)$ is continuous with respect to both variables, $t$ and $x$. In our case, the integrand $t^{-\theta} \Delta(t) \log ^{j} t$ is not continuous with respect to $t$, hence we cannot apply the theorem (Theorem 3.4 on p. 340 in [5]) directly in our present case. However, we stress that we can modify the proof of (1.6) described in [5] so as to be able to apply it. In fact, formula (1.6) is applicable to our case by using the first assertion of Theorem 1 and the properties of the integrand that have polynomial orders with respect to $t$.

As we have discussed above, the proof of Theorem 1 in [2] depends on the properties of integrands and deep theorems in analysis. In order to clarify the connection between the assertions of Theorem 1 and the properties of integral (1.1), we shall seek the new and elementary proof different from the previous one. In particular, we shall ask whether it is possible to deduce (1.5) without using (1.6) or not. Concerning this problem, we get the following.

THEOREM 2. The second assertion of Theorem 1 can be proved without applying the differentiation under the integral sign of the type (1.6). Actually, Theorem 1 can be proved by using the 'elementary' integral formulas concerning the Riemann zeta-and the periodic Bernoulli functions.

This theorem means that expression (1.5) is unrelated to the uniform convergence of integral (1.1) in the region $1<\Re \theta<5 / 4$.

In order to prove Theorem 2, we shall derive an asymptotic formula for integral $\int_{1}^{x} t^{-\theta} \Delta(t) \log ^{j} t d t$ with error terms uniformly in $x$. We note that the method used in the proof of this theorem is elementary, but a little complicated, repeating the decompositions and the combinations of the terms related with $\int_{1}^{x} t^{-\theta} \Delta(t) \log ^{j} t d t$. But the most important point is that we do not use any theoretically difficult things to derive representation (1.5) for $1<\Re \theta \leq 5 / 4$.

The analogous result to Theorem 1 in the case of the circle problem was discussed in [3]. For a natural number $n$, let $r(n)$ be the number of ways to write $n$ as the sum of 
two squares, and $P(x)$ be the error term in the circle problem defined by

$$
P(x)=\sum_{n \leq x} r(n)-\pi x .
$$

On this error function, we studied the properties of the integral

$$
\int_{1}^{\infty} t^{-\theta} P(t) \log ^{j} t d t
$$

and derived that this integral, convergent absolutely and uniformly on every compact subset in $\Re \theta>5 / 4$, is convergent uniformly on every compact subset in $1<\Re \theta \leq 5 / 4$, and is divergent for $\Re \theta \leq 1$. $^{*}$ In particular, the explicit representation of (1.7) for $\mathfrak{R} \theta>1$ is given by

$$
\begin{aligned}
\int_{1}^{\infty} t^{-\theta} P(t) \log ^{j} t d t= & 4 \sum_{m=0}^{j}\left(\begin{array}{c}
j \\
m
\end{array}\right) \frac{(-1)^{j-m} m !}{(\theta-1)^{m+1}}(\zeta(\theta-1) L(\theta-1, \chi))^{(j-m)} \\
& -\frac{\pi j !}{(\theta-2)^{j+1}}
\end{aligned}
$$

(cf. Theorem 1 and some formulas in Section 1 in [3]). The method used in [3] is the analogue of that used in the case of the divisor problem [2]; however, this case is more difficult than that of the divisor problem. It is because we have newly considered some identities related to the Dirichlet character $\chi(\bmod 4)$ adding the formulas used in [2], e.g. $\sum_{n \leq x} \chi(n) n^{1-\theta}$ or $\sum_{n \leq x} \chi(n) \int_{1}^{x / n} t^{-\theta} \psi(t) d t$ in the case of the circle problem, where $\psi(x)$ is the periodic Bernoulli function.

Similar to the case of the divisor problem, we also proved the convergence of integral (1.7) for $1<\Re \theta \leq 5 / 4$ without using the theorem of differentiation under the integral sign (1.6). We present this result as the following theorem.

THEOREM 3. Without applying differentiation under the integral sign of the type (1.6), we can prove that the integral (1.7) is convergent uniformly in the wider sense in the region $1<\Re \theta \leq 5 / 4$ and that it is represented by the right-hand side in (1.8).

We note that we have not yet determined the properties of (1.1) for general $k \geq 3$ completely, indeed in [2] we only proved that integral (1.1) is convergent absolutely for $\Re \theta>4 / 3$ for $k=3^{\dagger}$ and for $\Re \theta>A_{k}+1$ for $k \geq 4$, where

$$
A_{k}=\inf \left\{\alpha_{k} \mid \Delta_{k}(x)=O\left(x^{\alpha_{k}}\right) \text { for } x \geq 1\right\} .
$$

However, we have not yet discussed the properties of integral (1.1) for the other region of $\theta$, and it seems to be difficult to study these in such regions for general $k \geq 3$. The difficulties of these studies originate from that there is only a few information about the 'mean value formulas' for $\Delta_{k}(x)$. From this point, we should say that these studies would be quite difficult to obtain new results concerning these problems. But we believe

\footnotetext{
* Similar to the case of the Dirichlet divisor problem, the constant ' $5 / 4$ ' is the best-possible one that we can obtain by the method used in our argument.

${ }^{\dagger}$ We again note that the constant ' $4 / 3$ ' is the best-possible one, which we can obtain by the method used in our argument, since $A_{3} \geq 1 / 3$.
} 
that the method in [2] and developed in this paper would be useful in the study of these topics and we would discuss these topics elsewhere.

We remark that the referee of this paper pointed out two important remarks concerning the related problems discussed in paper [2] and the original version of this paper. We shall discuss these topics in Section 5.

The authors would like to express their sincere gratitude to the referee for his/her valuable comments.

2. Preliminaries. Throughout the paper, $\theta$ denotes a complex number, and we may assume that $1<\Re \theta \leq 5 / 4$ in the latter discussion. Function $\psi(x)$ denotes the periodic Bernoulli function defined by $\psi(x)=x-[x]-1 / 2$, where $[x]$ is the greatest integer not exceeding $x$.

In this section, we shall prepare some integral and summation formulas concerning the $\psi$ - and log-functions.

LEMMA 1. We have

$$
\int_{1}^{\infty} t^{-2} \psi(t) d t=\frac{1}{2}-\gamma
$$

Furthermore, let $s$ be a complex number for $\Re s>1$ with $s \neq 2$, and $j$ be a fixed nonnegative integer. We have

$$
\begin{aligned}
\int_{1}^{\infty} t^{-s} \psi(t) \log ^{j} t d t= & \frac{j !}{(s-2)^{j+1}}-\frac{j !}{2(s-1)^{j+1}} \\
& -j ! \sum_{n=0}^{j} \frac{(-1)^{n}}{n !}\left(\frac{1}{s-1}\right)^{j-n+1}(\zeta(s-1))^{(n)} .
\end{aligned}
$$

Proof. These are Lemma 3 and Lemma 4 in [2], respectively.

Note that assertions of this lemma, especially the second assertion, can be derived by employing differentiation under the integral sign. But we did not use (1.6) by means of Theorem 3.4 (p. 340) in [5] to prove this formula. It is because that the integral on the right-hand side is convergent absolutely, it is Lebesgue-integrable. We also note that the asymptotic formula of the partial integral $\int_{1}^{x} t^{-s} \psi(t) \log ^{j} t d t$ can be derived by applying Lemma 1 to the relation

$$
\int_{1}^{x} t^{-s} \psi(t) \log ^{j} t d t=\int_{1}^{\infty} t^{-s} \psi(t) \log ^{j} t d t+O\left(x^{-\Re i s} \log ^{j} x\right)
$$

for $\Re s>11^{\ddagger}$

LEMMA 2. For a complex number $s$ with $s \neq 1$ and a fixed non-negative integer $j$, we have

$$
\int_{1}^{x} t^{-s} \log ^{j} t d t=\frac{j !}{(s-1)^{j+1}}-x^{1-s} \sum_{m=0}^{j}\left(\begin{array}{c}
j \\
m
\end{array}\right) \frac{m !}{(s-1)^{m+1}} \log ^{j-m} x
$$

Proof. This is the formula (5.10) in [2].

${ }_{\ddagger}^{\ddagger}$ In fact, this formula is valid for $\Re s>0$ by using integration by parts and the estimate $\int_{1}^{x} \psi(t) d t=O(1)$. 
LEMMA 3. For $s \neq 2$ and a non-negative integer $j$, we have

$$
\begin{aligned}
\sum_{n \leq x} n^{1-s} \log ^{j} n= & \frac{j !}{(s-2)^{j+1}}-x^{2-s} \sum_{m=0}^{j}\left(\begin{array}{c}
j \\
m
\end{array}\right) \frac{m !}{(s-2)^{m+1}} \log ^{j-m} x-\psi(x) x^{1-s} \log ^{j} x \\
& +(1-s) \int_{1}^{x} t^{-s} \psi(t) \log ^{j} t d t+j \int_{1}^{x} t^{-s} \psi(t) \log ^{j-1} t d t+c_{j},
\end{aligned}
$$

where $c_{j}$ is the constant defined by $c_{0}=1 / 2$ and $c_{j}=0$ for $j \geq 1$.

Proof. This formula is proved by using the Euler-Maclaurin summation formula and by applying Lemma 2 .

In the study of integral (1.3) for $j \geq 1$, we need to apply the formulas for the partial integral $\int_{1}^{x} t^{-\theta} \Delta(t) d t$. We present the result concerning such formulas in the following lemma.

LeMma 4. For a sufficiently large $x$, we have

$$
\begin{aligned}
\int_{1}^{x} t^{-\theta} \Delta(t) d t= & \frac{1-2 \gamma}{2-\theta} x^{2-\theta}+\frac{1}{4(1-\theta)} x^{1-\theta}+\left\{\frac{2 \gamma}{2-\theta}+\frac{5 \theta-6}{4(1-\theta)(2-\theta)}\right\} \\
& -\frac{1}{2-\theta} x^{2-\theta} \int_{1}^{x} t^{-2} \psi(t) d t+\frac{\theta}{2(2-\theta)} \int_{1}^{x} t^{-\theta} \psi(t) d t \\
& -\sum_{n \leq x} n^{1-\theta} \int_{1}^{x / n} t^{-\theta} \psi(t) d t .
\end{aligned}
$$

Furthermore, an asymptotic formula for this integral can be written down as

$$
\int_{1}^{x} t^{-\theta} \Delta(t) d t=\frac{1}{\theta-1} \zeta^{2}(\theta-1)+\frac{2 \gamma}{2-\theta}+\frac{\theta-3}{(2-\theta)^{2}}+O\left(x^{1-\Re \theta} \log x\right)
$$

for $1<\Re \theta \leq 5 / 4$.

Proof. The first formula is derived from formulas (3.2) and (3.3) in [2] and the second one is formula (3.10) in [2].

3. Proof of Theorem 2. Hereafter, we may assume that $j$ is a fixed natural number. Let $x$ be a sufficiently large number. We start from the formula

$$
\int_{1}^{x} t^{-\theta} \Delta(t) \log ^{j} t d t=\log ^{j} x \int_{1}^{x} t^{-\theta} \Delta(t) d t-j \int_{1}^{x} \frac{\log ^{j-1} t}{t} \int_{1}^{t} u^{-\theta} \Delta(u) d u d t
$$

which is given by integration by parts. Substituting the first and second formulas in Lemma 4 into the innermost integral of the second integral and the first one on the 
right-hand side in (3.1) and interchanging integration and summation, we have

$$
\begin{aligned}
& \int_{1}^{x} t^{-\theta} \Delta(t) \log ^{j} t d t \\
&=\left\{\frac{\theta^{2}}{4(1-\theta)(2-\theta)^{2}}+\frac{1}{\theta-1} \zeta^{2}(\theta-1)\right\} \log ^{j} x-\frac{j}{4(1-\theta)} \int_{1}^{x} t^{-\theta} \log ^{j-1} t d t \\
&+\frac{(2 \gamma-1) j}{2-\theta} \int_{1}^{x} t^{1-\theta} \log ^{j-1} t d t+\frac{j}{2-\theta} \int_{1}^{x} t^{-2} \psi(t) \int_{t}^{x} u^{1-\theta} \log ^{j-1} u d u d t \\
& \\
& \quad-\frac{\theta j}{2(2-\theta)} \int_{1}^{x} t^{-\theta} \psi(t) \int_{t}^{x} u^{-1} \log ^{j-1} u d u d t \\
&+j \sum_{n \leq x} n^{1-\theta} \int_{n}^{x} t^{-1} \log ^{j-1} t \int_{1}^{t / n} u^{-\theta} \psi(u) d u d t+O\left(x^{1-\Re \theta} \log ^{j+1} x\right) .
\end{aligned}
$$

It is easy to see that

$$
\begin{aligned}
& j \sum_{n \leq x} n^{1-\theta} \int_{n}^{x} t^{-1} \log ^{j-1} t \int_{1}^{t / n} u^{-\theta} \psi(u) d u d t \\
& \quad=\log ^{j} x \sum_{n \leq x} n^{1-\theta} \int_{1}^{x / n} t^{-\theta} \psi(t) d t-\sum_{n \leq x} n^{1-\theta} \int_{1}^{x / n} t^{-\theta} \psi(t) \log ^{j}(n t) d t .
\end{aligned}
$$

We note that the first part on the right-hand side in the above has been treated in the previous paper [2]. Actually, we have seen that

$$
\begin{aligned}
\sum_{n \leq x} n^{1-\theta} \int_{1}^{x / n} t^{-\theta} \psi(t) d t \\
=(1-\theta)\left\{\frac{1}{\theta-2}-\frac{1}{2(\theta-1)}-\frac{1}{\theta-1} \zeta(\theta-1)\right\}^{2}+\frac{1}{2-\theta} x^{2-\theta} \int_{1}^{x} t^{-2} \psi(t) d t \\
\quad-\frac{\theta}{2(2-\theta)} \int_{1}^{x} t^{-\theta} \psi(t) d t+O\left(x^{1-\Re \theta} \log x\right)
\end{aligned}
$$

(see formula (3.4) and the last formula on p. 11 in [2]). Thus, we have by Lemma 1 that

$$
\begin{aligned}
\int_{1}^{x} t^{-\theta} \Delta(t) \log ^{j} t d t \\
=\frac{1-2 \gamma}{2(2-\theta)} x^{2-\theta} \log ^{j} x+\frac{(2 \gamma-1) j}{2(2-\theta)} \int_{1}^{x} t^{1-\theta} \log ^{j-1} t d t \\
\quad-\frac{j}{4(1-\theta)} \int_{1}^{x} t^{-\theta} \log ^{j-1} t d t-\frac{j}{2-\theta} \int_{1}^{x} t^{-2} \psi(t) \int_{1}^{t} u^{1-\theta} \log ^{j-1} u d u d t \\
\quad+\frac{\theta}{2(2-\theta)} \int_{1}^{x} t^{-\theta} \psi(t) \log ^{j} t d t-\sum_{n \leq x} n^{1-\theta} \int_{1}^{x / n} t^{-\theta} \psi(t) \log ^{j}(n t) d t \\
\quad+O\left(x^{1-\Re \theta} \log ^{j+1} x\right) .
\end{aligned}
$$

We consider the sixth part on the right-hand side in the above. By applying the binomial expansion to $\log ^{j}(n t)$ and interchanging the summations and integration, we 
have

$$
\begin{aligned}
& \sum_{n \leq x} n^{1-\theta} \int_{1}^{x / n} t^{-\theta} \psi(t) \log ^{j}(n t) d t \\
& \quad=\sum_{\nu=0}^{j}\left(\begin{array}{l}
j \\
v
\end{array}\right) \int_{1}^{x} t^{-\theta} \psi(t) \log ^{j-v} t \sum_{n \leq x / t} n^{1-\theta} \log ^{\nu} n d t .
\end{aligned}
$$

Then, we apply Lemma 3 to the right-hand side in (3.3) to obtain that this is written down as

$$
\begin{aligned}
= & \sum_{\nu=0}^{j}\left\{c_{\nu}+\frac{v !}{(\theta-2)^{\nu+1}}\right\}\left(\begin{array}{l}
j \\
v
\end{array}\right) \int_{1}^{x} t^{-\theta} \psi(t) \log ^{j-\nu} t d t \\
& -x^{2-\theta} \sum_{\nu=0}^{j}\left(\begin{array}{l}
j \\
v
\end{array}\right) \sum_{m=0}^{\nu}\left(\begin{array}{l}
v \\
m
\end{array}\right) \frac{m !}{(\theta-2)^{m+1}} \int_{1}^{x} t^{-2} \psi(t) \log ^{j-v} t \log ^{\nu-m}\left(\frac{x}{t}\right) d t \\
& +(1-\theta) \sum_{\nu=0}^{j}\left(\begin{array}{l}
j \\
v
\end{array}\right) \int_{1}^{x} t^{-\theta} \psi(t) \log ^{j-v} t \int_{1}^{x / t} u^{-\theta} \psi(u) \log ^{\nu} u d u d t \\
& +\sum_{\nu=1}^{j}\left(\begin{array}{l}
j \\
v
\end{array}\right) v \int_{1}^{x} t^{-\theta} \psi(t) \log ^{j-v} t \int_{1}^{x / t} u^{-\theta} \psi(u) \log ^{\nu-1} u d u d t+O\left(x^{1-\Re \theta} \log ^{j+1} x\right) .
\end{aligned}
$$

As for double integrals in the above, we have for non-negative integers $a$ and $b$ and a complex number $\theta$ with $\Re \theta>1$,

$$
\begin{aligned}
& \int_{1}^{x} t^{-\theta} \psi(t) \log ^{a} t \int_{1}^{x / t} u^{-\theta} \psi(u) \log ^{b} u d u d t \\
& \quad=\left(\int_{1}^{\infty} t^{-\theta} \psi(t) \log ^{a} t d t\right)\left(\int_{1}^{\infty} t^{-\theta} \psi(t) \log ^{b} t d t\right)+O\left(x^{1-\Re \theta} \log ^{a+b} x\right) .
\end{aligned}
$$

Furthermore, we can see by applying the binomial expansion once again that

$$
\begin{aligned}
& \int_{1}^{x} t^{-2} \psi(t) \log ^{j-v} t \log ^{\nu-m}\left(\frac{x}{t}\right) d t \\
& =\sum_{l=0}^{\nu-m}(-1)^{\nu-m-l}\left(\begin{array}{c}
v-m \\
l
\end{array}\right) \log ^{l} x \int_{1}^{x} t^{-2} \psi(t) \log ^{j-m-l} t d t
\end{aligned}
$$

Now we substitute all formulas derived above into (3.2). We note that formula

$$
j \sum_{n=0}^{j-1}\left(\begin{array}{c}
j-1 \\
n
\end{array}\right) n ! f(n)=\sum_{n=1}^{j}\left(\begin{array}{l}
j \\
n
\end{array}\right) n ! f(n-1)
$$


holds for $j \geq 1$ and any function $f$. Thereby by using Lemmas 1 and 2 with noting the remark below Lemma 1 , we obtain

$$
\begin{aligned}
\int_{1}^{x} t^{-\theta} & \Delta(t) \log ^{j} t d t \\
= & \frac{2 \gamma-1}{2} x^{2-\theta} \sum_{\nu=0}^{j}\left(\begin{array}{l}
j \\
v
\end{array}\right) \frac{v !}{(\theta-2)^{\nu+1}} \log ^{j-v} x+x^{2-\theta} \sum_{\nu=0}^{j} \frac{j !}{(j-v) !} \sum_{m=0}^{\nu} \frac{1}{(\theta-2)^{m+1}} \\
& \times \sum_{l=0}^{\nu-m} \frac{(-1)^{\nu-m-l}}{l !(v-m-l) !} \log ^{l} x \int_{1}^{\infty} t^{-2} \psi(t) \log ^{j-m-l} t d t \\
& -\frac{j}{2-\theta} \int_{1}^{\infty} t^{-2} \psi(t) \int_{1}^{t} u^{1-\theta} \log ^{j-1} u d u d t-\frac{\theta}{\theta-2} \int_{1}^{\infty} t^{-\theta} \psi(t) \log ^{j} t d t \\
& +(\theta-1) \sum_{\nu=0}^{j}\left(\begin{array}{l}
j \\
v
\end{array}\right)\left(\int_{1}^{\infty} t^{-\theta} \psi(t) \log ^{j-v} t d t\right)\left(\int_{1}^{\infty} t^{-\theta} \psi(t) \log ^{v} t d t\right) \\
& -\sum_{\nu=1}^{j}\left(\begin{array}{l}
j \\
v
\end{array}\right) v\left(\int_{1}^{\infty} t^{-\theta} \psi(t) \log ^{j-v} t d t\right)\left(\int_{1}^{\infty} t^{-\theta} \psi(t) \log ^{\nu-1} t d t\right) \\
& -\sum_{\nu=1}^{j}\left(\begin{array}{l}
j \\
v
\end{array}\right) \frac{v !}{(\theta-2)^{\nu+1}} \int_{1}^{\infty} t^{-\theta} \psi(t) \log ^{j-v} t d t+\frac{j !}{4(\theta-1)^{j+1}}-\frac{(2 \gamma-1) j !}{2(\theta-2)^{j+1}} \\
& +O\left(x^{1-\Re \theta \theta} \log ^{j+1} x\right)
\end{aligned}
$$

for $1<\Re \theta \leq 5 / 4$.

We transform the second part on the right-hand side in (3.5). Interchanging the summations, we see that this part is equal to

$$
j ! x^{2-\theta} \sum_{m=0}^{j} \frac{1}{(\theta-2)^{m+1}} \sum_{l=0}^{j-m} \frac{\log ^{l} x}{l !} \sum_{\nu=l+m}^{j} \frac{(-1)^{\nu-m-l}}{(j-v) !(\nu-m-l) !} \int_{1}^{\infty} t^{-2} \psi(t) \log ^{j-m-l} t d t .
$$

Furthermore, by applying the well-known formula

$$
\sum_{n=0}^{N}(-1)^{n}\left(\begin{array}{l}
N \\
n
\end{array}\right)= \begin{cases}1 & \text { if } N=0 \\
0 & \text { otherwise }\end{cases}
$$

to the innermost sum in the above and using Lemma 1, we can see that this is the negative of the first part on the right-hand side in (3.5). As for the third part on the right-hand side in (3.5), we have by Lemmas 1 and 2, and formula (3.4) that this is

$$
=\frac{(1-2 \gamma) j !}{2(\theta-2)^{j+1}}-\sum_{m=1}^{j}\left(\begin{array}{c}
j \\
m
\end{array}\right) \frac{m !}{(\theta-2)^{m+1}} \int_{1}^{\infty} t^{-\theta} \psi(t) \log ^{j-m} t d t
$$


The formula in Lemma 1 leads

$$
\begin{aligned}
& -\frac{2 v !}{(\theta-2)^{\nu+1}}+(\theta-1) \int_{1}^{\infty} t^{-\theta} \psi(t) \log ^{\nu} t d t-v \int_{1}^{\infty} t^{-\theta} \psi(t) \log ^{\nu-1} t d t \\
& =-\frac{\nu !}{(\theta-2)^{\nu+1}}-(-1)^{\nu}(\zeta(\theta-1))^{(\nu)}
\end{aligned}
$$

for $v \geq 1$.

Consequently, by tending parameter $x$ to infinity we obtain

$$
\begin{aligned}
& \int_{1}^{\infty} t^{-\theta} \Delta(t) \log ^{j} t d t \\
& =-\sum_{\nu=0}^{j}\left(\begin{array}{l}
j \\
v
\end{array}\right)\left\{\frac{v !}{(\theta-2)^{v+1}}+(-1)^{\nu}(\zeta(\theta-1))^{(v)}\right\} \int_{1}^{\infty} t^{-\theta} \psi(t) \log ^{j-v} t d t \\
& -\frac{1}{2} \int_{1}^{\infty} t^{-\theta} \psi(t) \log ^{j} t d t+\frac{j !}{4(\theta-1)^{j+1}}+\frac{(1-2 \gamma) j !}{(\theta-2)^{j+1}}
\end{aligned}
$$

for $1<\Re \theta \leq 5 / 4$.

Now we will see that the main term in the above accords with that in formula (1.5). We denote the first part on the right-hand side in the above by $-S$, and we treat the transformation of it. By Lemma 1, we get

$$
\begin{aligned}
S= & \frac{(j+1) !}{(\theta-2)^{j+2}}-\frac{1}{2} j ! \sum_{\nu=0}^{j} \frac{1}{(\theta-2)^{\nu+1}(\theta-1)^{j-\nu+1}} \\
& +\sum_{\nu=0}^{j}\left(\begin{array}{l}
j \\
v
\end{array}\right) \frac{(-1)^{\nu}(j-v) !}{(\theta-2)^{j-v+1}}(\zeta(\theta-1))^{(\nu)}-\frac{1}{2} \sum_{\nu=0}^{j}\left(\begin{array}{l}
j \\
v
\end{array}\right) \frac{(-1)^{\nu}(j-v) !}{(\theta-1)^{j-\nu+1}}(\zeta(\theta-1))^{(\nu)} \\
& -j ! \sum_{\nu=0}^{j} \frac{1}{(\theta-2)^{\nu+1}} \sum_{n=0}^{j-v} \frac{(-1)^{n}}{n !} \frac{1}{(\theta-1)^{j-\nu-n+1}}(\zeta(\theta-1))^{(n)} \\
& -\sum_{\nu=0}^{j}\left(\begin{array}{l}
j \\
v
\end{array}\right)(-1)^{\nu}(j-v) !(\zeta(\theta-1))^{(\nu)} \sum_{n=0}^{j-v} \frac{(-1)^{n}}{n !} \frac{1}{(\theta-1)^{j-v-n+1}}(\zeta(\theta-1))^{(n)} \\
= & \frac{(j+1) !}{(\theta-2)^{j+2}}+\sum_{l=1}^{5} S_{l},
\end{aligned}
$$

say. We can easily get

$$
S_{1}=-\frac{1}{2} j !\left\{\frac{1}{(\theta-2)^{j+1}}-\frac{1}{(\theta-1)^{j+1}}\right\}
$$

since

$$
\sum_{n=0}^{a}\left(\frac{1}{\theta-2}\right)^{n+1}\left(\frac{1}{\theta-1}\right)^{a-n+1}=\frac{1}{(\theta-2)^{a+1}}-\frac{1}{(\theta-1)^{a+1}}
$$


for a non-negative integer $a$. Similarly, in $S_{4}$, by interchanging the summations and using (3.7) we have

$$
\begin{aligned}
S_{4} & =-j ! \sum_{n=0}^{j} \frac{(-1)^{n}}{n !}(\zeta(\theta-1))^{(n)} \sum_{\nu=0}^{j-n} \frac{1}{(\theta-2)^{\nu+1}} \frac{1}{(\theta-1)^{j-n-\nu+1}} \\
& =-j ! \sum_{n=0}^{j} \frac{(-1)^{n}}{n !(\theta-2)^{j-n+1}}(\zeta(\theta-1))^{(n)}+j ! \sum_{n=0}^{j} \frac{(-1)^{n}}{(\theta-1)^{j-n+1} n !}(\zeta(\theta-1))^{(n)},
\end{aligned}
$$

thus $S_{4}=-S_{2}-2 S_{3}$.

For $S_{3}$, we get by Lemma 1 directly that

$$
S_{3}=\frac{1}{2} \int_{1}^{\infty} t^{-\theta} \psi(t) \log ^{j} t d t-\frac{j !}{2(\theta-2)^{j+1}}+\frac{j !}{4(\theta-1)^{j+1}} .
$$

Rearranging the parameter of the summation with respect to $n$ as $m=j-v-n$ and interchanging the double sums in $S_{5}$, we have

$$
\begin{aligned}
S_{5} & =-j ! \sum_{\nu=0}^{j} \frac{(-1)^{\nu}}{\nu !}(\zeta(\theta-1))^{(\nu)} \sum_{m=0}^{j-v} \frac{(-1)^{j-m-\nu}}{(j-m-v) !} \frac{1}{(\theta-1)^{m+1}}(\zeta(\theta-1))^{(j-m-\nu)} \\
& =-j ! \sum_{m=0}^{j} \frac{(-1)^{j-m}}{(\theta-1)^{m+1}} \sum_{\nu=0}^{j-m} \frac{1}{\nu !(j-m-\nu) !}(\zeta(\theta-1))^{(\nu)}(\zeta(\theta-1))^{(j-m-\nu)}
\end{aligned}
$$

Finally, by applying the Leibniz rule to the innermost sum in the last equation in the above we have

$$
S_{5}=-\sum_{m=0}^{j}\left(\begin{array}{l}
j \\
m
\end{array}\right) \frac{m !(-1)^{j-m}}{(\theta-1)^{m+1}}\left(\zeta^{2}(\theta-1)\right)^{(j-m)} .
$$

Therefore, we obtain

$$
\begin{aligned}
S= & -\sum_{m=0}^{j}\left(\begin{array}{c}
j \\
m
\end{array}\right) \frac{m !(-1)^{j-m}}{(\theta-1)^{m+1}}\left(\zeta^{2}(\theta-1)\right)^{(j-m)}-\frac{1}{2} \int_{1}^{\infty} t^{-\theta} \psi(t) \log ^{j} t d t \\
& +\frac{j !}{4(\theta-1)^{j+1}}+\frac{(j+1) !}{(\theta-2)^{j+2}} .
\end{aligned}
$$

By substituting this formula into (3.6), we obtain representation (1.5) for $1<\Re \theta \leq 5 / 4$. This completes the alternative proof of Theorem 1 for $j \geq 1$, thus the proof of Theorem 2 is complete.

4. Proof of Theorem 3. Based on the methods used in Section 3, we shall consider the alternative approach for formula (1.8) for $j \geq 1$. However, the proof in this section is much closed to that used in the previous section. Thus, we will give only a sketch of this proof. 
We start from the identity

$$
\begin{aligned}
\int_{1}^{x} t^{-\theta} P(t) d t= & \frac{4}{2-\theta} x^{2-\theta} \sum_{n \leq x} \chi(n) n^{-1}+\frac{2 \theta}{(1-\theta)(2-\theta)} \sum_{n \leq x} \chi(n) n^{1-\theta} \\
& -\frac{2}{1-\theta} x^{1-\theta} \sum_{n \leq x} \chi(n)-\frac{\pi}{2-\theta} x^{2-\theta}+\frac{\pi}{2-\theta} \\
& -4 \sum_{n \leq x} \chi(n) n^{1-\theta} \int_{1}^{x / n} t^{-\theta} \psi(t) d t
\end{aligned}
$$

and the asymptotic formula

$$
\int_{1}^{x} t^{-\theta} P(t) d t=\frac{4}{\theta-1} \zeta(\theta-1) L(\theta-1, \chi)-\frac{\pi}{\theta-2}+O\left(x^{1-\Re \theta} \log x\right)
$$

(cf. the formulas (2.3) and (2.5) in [3], respectively). Furthermore, we shall use the asymptotic formula

$$
\sum_{n \leq y} \chi(n) n^{-s} \log ^{\nu} n=(-1)^{\nu}(L(s, \chi))^{(\nu)}+O\left(y^{-\Re s} \log ^{\nu} y\right)
$$

frequently, where $v$ is a non-negative integer and $s$ is a complex number with $\Re s>0 . \S$

Then we have, by applying integration by parts, these two formulas, the second formula in Lemma 1 and Lemma 2 that

$$
\begin{aligned}
\int_{1}^{x} t^{-\theta} P(t) \log ^{j} t d t= & -\frac{\pi j !}{(\theta-2)^{j+1}}+4 \sum_{\nu=0}^{j}\left(\begin{array}{l}
j \\
v
\end{array}\right) \frac{(-1)^{j-v} v !}{(\theta-2)^{\nu+1}}(L(\theta-1, \chi))^{(j-v)} \\
& -2 \sum_{\nu=0}^{j}\left(\begin{array}{l}
j \\
v
\end{array}\right) \frac{(-1)^{j-v} v !}{(\theta-1)^{\nu+1}}(L(\theta-1, \chi))^{(j-v)} \\
& -4 \sum_{n \leq x} \chi(n) n^{1-\theta} \int_{1}^{x / n} t^{-\theta} \psi(t) \log ^{j}(n t) d t+O\left(x^{1-\Re \theta} \log ^{j+1} x\right) .
\end{aligned}
$$

In the second term from the last on the right-hand side, we apply the binomial expansion and the second formula in Lemma 1 that this part is equal to

$$
\begin{aligned}
& -4 \sum_{m=0}^{j}\left(\begin{array}{c}
j \\
m
\end{array}\right) \frac{(-1)^{j-m} m !}{(\theta-2)^{m+1}}(L(\theta-1, \chi))^{(j-m)} \\
& +4 \sum_{m=0}^{j}\left(\begin{array}{c}
j \\
m
\end{array}\right)(-1)^{j-m} m !(L(\theta-1, \chi))^{(j-m)} \sum_{n=0}^{m} \frac{(-1)^{n}}{n !(\theta-1)^{m-n+1}}(\zeta(\theta-1))^{(n)} \\
& +2 \sum_{m=0}^{j}\left(\begin{array}{c}
j \\
m
\end{array}\right) \frac{(-1)^{j-m} m !}{(\theta-1)^{m+1}}(L(\theta-1, \chi))^{(j-m)}+O\left(x^{1-\Re \theta} \log ^{j+1} x\right) .
\end{aligned}
$$

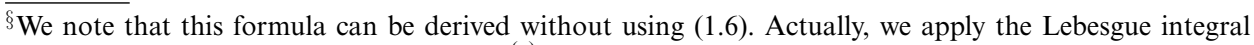
theorem (Theorem 10.39 in [1] $)$ to $(L(s, \chi))^{(v)}$ for $\Re s>1$. Then we prove this formula for $\Re s>1$ and thus for $\Re s>0$ by analytic continuation of $(L(s, \chi))^{(v)}$. 
Thereby substituting this formula into (4.1), we obtain

$$
\begin{aligned}
& \int_{1}^{x} t^{-\theta} P(t) \log ^{j} t d t \\
& =4 \sum_{m=0}^{j}\left(\begin{array}{c}
j \\
m
\end{array}\right)(-1)^{j-m} m !(L(\theta-1, \chi))^{(j-m)} \sum_{n=0}^{m} \frac{(-1)^{n}}{n !(\theta-1)^{m-n+1}}(\zeta(\theta-1))^{(n)} \\
& \quad-\frac{\pi j !}{(\theta-2)^{j+1}}+O\left(x^{1-\Re(\theta} \log ^{j+1} x\right) .
\end{aligned}
$$

Furthermore, we treat the transformation of the first term on the right-hand side in (4.2). By interchanging the summations and rearranging the parameter of the summation with respect to $m$, we see that this part is rewritten as

$$
4 \sum_{n=0}^{j} \sum_{m=0}^{j-n}\left(\begin{array}{c}
j \\
m
\end{array}\right)\left(\begin{array}{c}
j-m \\
n
\end{array}\right) \frac{(-1)^{j-m} m !}{(\theta-1)^{m+1}}(\zeta(\theta-1))^{(n)}(L(\theta-1, \chi))^{(j-n-m)},
$$

since

$$
(m+n) !\left(\begin{array}{c}
j \\
m+n
\end{array}\right)=m ! n !\left(\begin{array}{c}
j \\
m
\end{array}\right)\left(\begin{array}{c}
j-m \\
n
\end{array}\right) .
$$

Hence, by interchanging the summations again and applying the Leibniz rule to the innermost sum, we obtain

$$
\begin{aligned}
\int_{1}^{x} t^{-\theta} P(t) \log ^{j} t d t= & 4 \sum_{m=0}^{j}\left(\begin{array}{c}
j \\
m
\end{array}\right) \frac{(-1)^{j-m} m !}{(\theta-1)^{m+1}}(\zeta(\theta-1) L(\theta-1, \chi))^{(j-m)} \\
& -\frac{\pi j !}{(\theta-2)^{j+1}}+O\left(x^{1-\Re \theta} \log ^{j+1} x\right) .
\end{aligned}
$$

This completes the proof of Theorem 3 .

5. Addendum. In this section, we shall discuss two points that the anonymous referee kindly informed us.

Let us put

$$
\widetilde{\Delta}(x)=\sum_{n \leq x}^{\prime} d(n)-x(\log x+2 \gamma-1)-\frac{1}{4},
$$

where symbol $\sum_{n<x}^{\prime}$ means that the last term is to be halved if $x$ is an integer. This definition of the error term is also used in the theory of the divisor problem, and we see that this function has the same and similar properties of $\Delta(x)$. For instance, we see that these two functions have the same upper and the lower bounds and that the formulas

$$
\int_{1}^{x} \Delta(t) d t=\frac{1}{4} x+O\left(x^{3 / 4}\right) \text { and } \quad \widetilde{\Delta}(x)=\int_{1}^{x} \Delta(t) d t-\frac{1}{4} x=O\left(x^{3 / 4}\right) .
$$

hold for $x \geq 1$.

Now we shall consider the explicit representations of the integral related to $\widetilde{\Delta}(x)$, namely, we shall study the convergence of the integral $\int_{1}^{\infty} t^{-\theta} \widetilde{\Delta}(t) d t$. By the definition 
of $\widetilde{\Delta}(x)$, we can see that

$$
\int_{1}^{x} t^{-\theta} \tilde{\Delta}(t) d t=\int_{1}^{x} t^{-\theta} \Delta(t) d t+ \begin{cases}-4^{-1} \log x & \text { if } \theta=1, \\ 4^{-1}(1-\theta)^{-1}\left(1-x^{1-\theta}\right) & \text { otherwise. }\end{cases}
$$

Thus, combining this formula with relation (2.1) in [2] we obtain

$$
\int_{1}^{x} t^{-\theta} \widetilde{\Delta}(t) d t=\left\{\begin{array}{ll}
\widetilde{C}(\theta)+O\left(x^{1-\Re \theta}\right) & \text { if } \Re \theta>3 / 4 \\
O\left(x^{3 / 4-\Re \theta}\right)+O(\log x) & \text { if } \Re \theta \leq 3 / 4
\end{array},\right.
$$

where $\widetilde{C}(\theta)$ is the constant depending only on $\theta$, especially, this can be written down explicitly as

$$
\widetilde{C}(\theta)=\theta \int_{1}^{\infty} t^{-\theta} \Delta_{1}(t) d t
$$

for $\Re \theta>3 / 4$ with $\theta \neq 1$. Here $\Delta_{1}(x)$ is the error function defined by $\Delta_{1}(x)=\int_{1}^{x} \widetilde{\Delta}(t) d t$, thus $\Delta_{1}(x)=\int_{1}^{x} \Delta(t) d t-\frac{1}{4} x$. (We note that the mean value result of $\Delta(x)$ mentioned above gives the estimate $\Delta_{1}(x)=O\left(x^{3 / 4}\right)$ obviously. $)$

Formula (5.1) states that the improper integral $\int_{1}^{\infty} t^{-\theta} \widetilde{\Delta}(t) d t$ is convergent absolutely and uniformly for $\Re \theta>5 / 4$ as in the case of $\int_{1}^{\infty} t^{-\theta} \Delta(t) d t$. Furthermore, this integral can be convergent uniformly on every compact subset for $3 / 4<\Re \theta \leq 5 / 4$, although the integral related to $\Delta(x)$ is convergent uniformly for the region $1<\Re \theta \leq$ $5 / 4$. However, integral $\int_{1}^{\infty} t^{-\theta} \widetilde{\Delta}(t) d t$ is not known to be convergent or divergent for $\Re \theta \leq 3 / 4$ at present. In order to prove it, we should improve the lower formula in the line (5.2), thus we should improve the estimate of the integral $\int_{1}^{x} t^{-\theta} \psi(t) \psi(x / t) d t$ obtained in Lemma 2 in [2]. This problem seems to be very interesting, so we shall return to this topic at another occasion.

Now we shall discuss other properties of integral (1.1) in the region $1<\Re \theta \leq 5 / 4$. In our previous paper [2], we conjectured that this integral is not absolutely convergent, i.e.

$$
\int_{1}^{\infty} t^{-\Re \theta}|\Delta(t)| \log ^{j} t d t=\infty
$$

for any complex number $\theta$ with $1<\Re \theta \leq 5 / 4$ and every non-negative integer $j$. It is pointed out by the referee that this conjecture holds true.

We shall give proof of (5.3) provided by the referee. The proof depends on the results of Heath-Brown and Tsang [4]. To prove (5.3), it is enough to consider integral

$$
\int_{X}^{2 X} t^{-a}|\Delta(t)| \log ^{j} t d t
$$

for $1<a \leq 5 / 4$. Let $X<x \leq 2 X$. By Theorem 2 in [4], we have $|\Delta(x)| \gg X^{1 / 4}$ for many disjoint intervals $I \subset[X, 2 X]$ of length $\gg \sqrt{X}(\log X)^{-5}$. The number of such intervals is $\gg \sqrt{X}(\log X)^{5}$. Consequently, for $a \leq 5 / 4$, we have

$$
\begin{aligned}
& \int_{X}^{2 X} t^{-a}|\Delta(t)| \log ^{j} t d t \gg X^{-a+1 / 4} \log ^{j} X \times \sqrt{X}(\log X)^{-5} \times \sqrt{X}(\log X)^{5} \\
& \gg X^{5 / 4-a} \log ^{j} X
\end{aligned}
$$

from which the conjecture (5.3) follows. 


\section{REFERENCES}

1. T. M. Apostol, Mathematical analysis, 2nd edn. (Addison-Wesley, Reading, MA, 1974).

2. J. Furuya and Y. Tanigawa, Explicit representations of the integral containing the error term in the divisor problem, Acta Math. Hungar. 129(1-2) (2010), 24-46.

3. J. Furuya and Y. Tanigawa, On the integrals containing the error term in the circle problem, preprint.

4. D. R. Heath-Brown and K. M. Tsang, Sign changes of $E(T), \Delta(x)$, and $P(x), J$. Num. Th. 49 (1994), 73-83.

5. S. Lang, Undergraduate analysis, 2nd Edn. (Undergraduate Texts in Mathematics, Springer-Verlag, New York, 1997).

6. A. F. Lavrik, M. I. Israilov and Ž. Edgorov, On integrals containing the error term in the divisor problem, Acta Arith. 37 (1980), 381-389. (in Russian)

7. R. Sitaramachandrarao, An integral involving the remainder term in the Piltz divisor problem, Acta Arith. 48(1) (1987), 89-92. 